\title{
SUR UNE CONJ ECTURE DE D. G. KENDALL CONCERNANT LA CELLULE DE CROFTON DU PLAN ET SUR SA CONTREPARTIE BROWNIENNE
}

Introduction. Soit $P=\left\{x_{n}, n \geq 1\right\}$ une mesure de Poisson aléatoire dans le plan $R^{2}$, de mesure d'intensité

$$
\mu(A)=E \operatorname{card}(A \cap P)=\frac{1}{2} \int_{-\infty}^{+\infty} \int_{0}^{2 \pi} 1_{A}(\sigma, r) d \sigma d r, \quad A \in B\left(R^{2}\right)
$$

et soit $H=\{H(x), x \in P\}$ l'ensemble des droites polaires associées, (communément connu sous le nom de Processus poissonien de droites). L'ensemble $H$ partitionne l'espace $\mathrm{R}^{2}$ en domaines polygonaux convexes (aléatoires) constituant la mosaïque poissonienne du plan [10]. En 1945, le physicien Goudsmit [8] a été amené à étudier les propriétés de leurs structures géométriques en calculant notamment, par une ingénieuse approche heuristique, les deux premiers moments empiriques de la mesure d'aire de ces domaines. Indépendamment de ce travail, le statisticien anglais Kendall a exploité, dans les années 1940/1945, le processus poissonien de droites comme modèle statistique des fibres du papier-les polygones aléatoires modélisant les interstices entre les fibres.

En 1964, Miles a débuté toute une série de travaux (s'étalant jusqu'en 1995) s'appuyant sur la nature ergodique du processus de droites $H$ et plus particulierement sur le fait que l'ensemble $H$ est invariant, en loi, par rotation et par translation (c'est-à-dire la mosaïque est homogène et isotrope). Ces résultats sont exposés dans [16]-[19]. Signalons encore les travaux de Cowan [2], [3], Matheron [15] et Tanner [24], [25], les simulations effectuées par Solomon [22] et George [5] et le récent travail de Paroux [20] obtenant, dans ce contexte, des théorèmes centraux limites.

Received November 1997; revised May 1998.

AMS 1991 subject classifications. Primary 60D05, 60 65; secondary 35P20, 52A22, 60F 10.

Key words and phrases. Poisson line process, Crofton cell, random polygons, perimeter of the convex hull of Brownian motion, eigenvalues. 
En dépit de ces recherches, la connaissance des lois des caractéristiques géométriques fondamentales que sont l'aire $V$, le périmètre $L$ ou le nombre de sommets $S$, de ces domaines, a peu progressé. On ne connait les valeurs exactes que des deux et parfois des trois premiers moments empiriques (on sait par exemple que $\tilde{E} V=1 / \pi, \tilde{E} L=2$ et $\tilde{E} S=4$ ) mais on manque d'informations sur les lois de ces caractéristiques. Plus particulièrement, la conjecture énoncée par Kendall (voir le mot d'introduction à l'ouvrage [23]) dans les années quarante, sel on laquelle la "forme" du domaine $\mathrm{D}_{0}$ contenant l'origine (la cellule de Crofton) ne serait pas très éloignée (dans un sens à préciser), Iorsque son aire $\mathrm{V}_{0}$ est "grande," de celle d'un disque, n'a pas été résolue. En 1995, Miles [19] a proposé plusieurs formulations possibles de cette conjecture en les étayant par des arguments de nature heuristique.

Dans le présent travail, nous décrivons précisément, le comportement asymptotique, lorsque $t \rightarrow+\infty$, de:

1. La probabilité $\mathrm{P}\left(\mathrm{V}_{0} \geq \mathrm{t}\right)$ relative à la cellule de Crofton;

2. La probabilité empirique $\tilde{P}\{V \geq t\}$

en démontrant que

$$
\lim _{\mathrm{t} \rightarrow+\infty} \mathrm{t}^{-1 / 2} \log \mathrm{P}\left\{\mathrm{V}_{0} \geq \mathrm{t}\right\}=\lim _{\mathrm{t} \rightarrow+\infty} \mathrm{t}^{-1 / 2} \log \tilde{P}\{\mathrm{~V} \geq \mathrm{t}\}=-2 \sqrt{\pi}
$$

Ces résultats nous permettent de montrer que la transformée de Laplace de la loi de la première valeur propre du Laplacien (pour le problème de Dirichlet) du domaine $D_{0}$ a le même comportement asymptotique, à l'infini, que la transformée de Laplace de la loi de la première valeur propre du Laplacien de la boule inscrite dans $D_{0}$, et que ce résultat reste valable en remplaçant la cellule de Crofton $D_{0}$ par le "polygone convexe empirique" D. La première valeur propre du Laplacien d'un domaine étant d'autant plus petite que le domaine est "grand," ce résultat fournit un élément de réponse positif (et rigoureux) à la conjecture de Kendall. Nous faisons ensuite appel à la relation (voir [6])

$$
\Phi(t)=\left(1 / 4 \pi^{2} t\right) E \exp \left(-\sqrt{2 t} Y_{0}\right), \quad t>0,
$$

reliant la fonction spectrale empirique $\Phi(\mathrm{t}), \mathrm{t}>0$, de la mosaïque poissonienne à la transformée de Laplace de la loi du périmètre $Y_{0}$ de l'enveloppe convexe de la trajectoire du pont brownien plan (relatif à l'intervalle de temps $[0,1])$ pour établir que

$$
\lim _{\mathrm{t} \rightarrow+\infty} \mathrm{t}^{-1 / 3} \log E \exp \left(-\sqrt{2 \mathrm{t}} \mathrm{Y}_{0}\right)=-\left(27 \pi^{2} \mathrm{j}_{0}^{2}\right)^{1 / 3},
$$

où $j_{0}$ est la première racine positive nonnulle de la fonction de Bessel $\mathrm{J} 0$. Ce dernier résultat est à rapprocher du comportement asymptotique du volume de la saucisse de Wiener (voir par exemple [4]).

Nous montrons que ce dernier résultat reste inchangé en remplaçant $Y_{0}$ par le périmètre $Y$ de l'enveloppe convexe du mouvement brownien plan puis nous en déduisons que la forme de l'enveloppe convexe du mouvement brownien plan, relatif à l'intervalle de temps $[0,1]$ et s'écartant peu, dans ce 
laps de temps, de son point de départ, et "proche" (dans un sens que nous précisons) de celle d'un disque. Cette propriété géométrique constitue la contrepartie brownienne de la conjecture de Kendall. Signalons encore que ces résultats peuvent probablement s'étendre au cas des mosaïques poissoniennes de l'espace euclidien $R^{d}, d \geq 3$, le rôle du périmètre, pour ce qui est des fonctionnelles $Y_{0}$ et $Y$, étant tenu par le diamètre moyen (ou, si l'on préfère, par le volume intrinsèque d'ordre un) des enveloppes convexes des trajectoires; nous y reviendrons dans un prochain travail.

Les résultats principaux de ce travail ont été annoncés dans la note [7].

1. Préliminaires. Dans ce paragraphe, nous rappelons quelques résultats connus des mosaïques poissoniennes de l'espace euclidien $R^{d}, d \geq 2$, qui vont nous être utiles par la suite. Pour une information plus exhaustive on pourra se reporter à [6], [10], [18] et [22].

A. La mosaïque poissonienne Soit $P=\left\{x_{n}, n \geq 1\right\}$ une mesure de Poisson aléatoire (définie sur un espace de probabilité $(\Omega, \Sigma, P)$ ) dans l'espace euclidien $R^{d}, d \geq 2$, de mesure d'intensité

$$
\begin{aligned}
\mu(A) & =E \operatorname{card}(A \cap P) \\
& =\left(\kappa_{d} / 2\right) \int_{R}\left\{\int_{s(d)} 1_{A}(\sigma, r) d \sigma\right\} d r, \quad A \in B\left(R^{d}\right),
\end{aligned}
$$

où on a désigné par $\mathrm{s}(\mathrm{d})$ la sphère unité de l'espace euclidien $\mathrm{R}^{\mathrm{d}}$, par $\kappa_{d} \mathrm{~d} \sigma$, $\kappa_{d}=\mathrm{d} \pi^{\mathrm{d} / 2} / \Gamma(\mathrm{d} / 2+1)$, sa mesure d'aire (non-normalisée) et par $1_{A}(\sigma, r)$ la fonction indicatrice de l'ensemble borélien $A$. Pour tout point $x \in R^{d}$, soit $\mathrm{H}(\mathrm{x})=\left\{\mathrm{y} \in \mathrm{R}^{\mathrm{d}} ;\langle\mathrm{y}-\mathrm{x}, \mathrm{x}\rangle=0\right.$ \} I'hyperplan polaire associé (en notant par $\langle\cdot, \cdot\rangle$ le produit scalaire de l'espace euclidien $R^{d}$ ). L'ensemble $H=\{H(x)$, $\mathrm{X} \in \mathrm{P}$ \} est connu sous le nom de processus poissonien d'hyperplans de l'espace $\mathrm{R}^{\mathrm{d}}$. Il est invariant, en loi, par les isométries de l'espace $\mathrm{R}^{\mathrm{d}}$ et partitionne ce dernier en polyèdres convexes (aléatoires).

B. La cellule de Crofton $D_{0}$. Fixons $x \in R^{d}$ et pour tout $\omega \in \Omega$, désignons par $D(x, \omega)$ le polyèdre contenant le point $x$. En fait, $x \in R^{d}$ et $\omega \in \Omega$ étant fixés, l'ensemble $\mathrm{D}(\mathrm{x}, \omega)$ n'est bien défini que si $\mathrm{x}$ n'appartient pas à la réunion des hyperplans de l'ensemble $H(\omega)$. Cela étant, le point $x$ étant fixé, la probabilité pour qu'il appartienne à l'un des hyperplans de $H$ est évidemment nulle et, de ce fait, $D(x, \omega)$ est bien défini pour tout $\omega \in \Omega \backslash N_{x}$, $P\left(N_{x}\right)=0$. Dans ce qui suit on désignera par $D_{0}(\omega)$ le polyèdre convexe contenant I'origine, c'est-à-dire, suivant la terminologie de Kendall [23], la cellule ce Crofton. Pour tout ensemble borélien $A \in B\left(R^{d}\right)$, notons par $\hat{A}$ son enveloppe convexe puis par

$$
\mathrm{b}(\mathrm{A})=2 \kappa_{\mathrm{d}} \int_{\mathrm{s}(\mathrm{d})} \sup _{\mathrm{x} \in \mathrm{A}}\langle\sigma, \mathrm{x}\rangle \mathrm{d} \sigma,
$$

le diamètre moyen de $A$. Rappelons qu'en dimension $d=2,(1 / 2) b(A)$ coïncide, en vertu d'un résultat classique de Cauchy, avec le périmètre de $\hat{A}$ (Voir [1]). 
Dans ce qui suit nous ferons appel à la propriété fondamentale suivante (voir [6] et [15]).

Lemme 1. Soit $A \in B\left(R^{d}\right)$ un ensemble borélien contenant l'origine et $\hat{A}$ son enveloppe convexe On a alors

$$
P\left\{D_{0} \supset A\right\}=P\left\{D_{0} \supset \hat{A}\right\}=\exp [-(1 / 2) b(A)] .
$$

C. Les distributions empiriques. Considérons maintenant la boule ouverte $B(R)=\left\{x \in R^{d} ;\|x\|<R\right\}$ centrée à l'origine et de rayon $R>0$ puis les polyèdres convexes $D_{i}, i=1, \ldots, N_{R}$, inclus dans $B(R)$. Soit $X$ une fonctionnelle opérant sur les polyèdres convexes de l'espace $R^{d}$ et invariante par translation et soit $\left(1 / N_{R}\right) \sum_{i=1}^{N_{R}} X\left(D_{i}\right), R>0$, la moyenne associée. On montre alors, grâce à la structure ergodique du processus H (et avec [26]), que sous des conditions raisonnables de mesurabilité, la moyenne empirique de $\mathrm{X}$, c'est-à-dire la limite presque-sûre,

$$
\tilde{E} X=\lim _{R \rightarrow+\infty}\left(1 / N_{R}\right) \sum_{i=1}^{N_{R}} X\left(D_{i}\right)
$$

existe et que l'on a

$$
\tilde{E} X=\left\{E\left(1 / V_{0}\right)\right\}^{-1} E\left\{X\left(D_{0}\right) / V_{0}\right\},
$$

où $V_{0}=\int_{D_{0}} d x$ est le volume de la cellule de Crofton. En particulier,

$$
\tilde{P}\{X \leq t\}=\lim _{R \rightarrow+\infty}\left(1 / N_{R}\right) \sum_{i=1}^{N_{R}} 1_{[0, t]}\left(X\left(D_{i}\right)\right)
$$

est, par définition, la fonction de répartition empirique de la fonctionnelle $\mathrm{X}$. Plus précisément, on dispose du résultat suivant (voir [2] et [6]).

Lemme 2. (i) Supposons que l'application $\omega \rightarrow\left\{1 / \mathrm{V}_{0}(\omega)\right\} \times\left(\mathrm{D}_{0}(\omega)\right)$ soit une variable aléatoire On a alors, presquesûrement, pour tout $t \geq 0$ fixé,

$$
\begin{aligned}
\tilde{P}\{X \leq t\} & =\lim _{R \rightarrow+\infty}\left(1 / N_{R}\right) \sum_{i=1}^{N_{R}} 1_{[0, t]}\left(X\left(D_{i}\right)\right) \\
& =\left\{E\left(1_{[0, t]}\right)\left(X\left(D_{0}\right)\right) / V_{0}\right\}\left\{E\left(1 / V_{0}\right)\right\}^{-1} .
\end{aligned}
$$

(ii) Supposons de plus que I'on ait $E\left(\left|X\left(D_{0}\right) / V_{0}\right|\right)<+\infty$. On a alors pour presquetout $\omega \in \Omega$,

$$
\tilde{E} X=\lim _{R \rightarrow+\infty}\left(1 / N_{R}\right) \sum_{i=1}^{N_{R}} X\left(D_{i}\right)
$$

$$
\begin{aligned}
& =\lim _{R \rightarrow+\infty}\left(1 / N_{R}\right) \int_{B(R)}\{X(D(x, \omega)) / V(x, \omega)\} d x \\
& =\left\{E\left(X\left(D_{0}\right) / V_{0}\right\}\left\{E\left(1 / V_{0}\right)\right\}^{-1} d t=\left\{E\left(X\left(D_{0}\right) / V_{0}\right)\right\}\left\{E\left(1 / V_{0}\right)\right\}^{-1} .\right.
\end{aligned}
$$


En prenant pour $\mathrm{X}$ la mesure d'aire du polyèdre convexe, on obtient

$$
\tilde{E} V^{n}=\left\{E V_{0}^{n-1}\right\}\left\{E\left(1 / V_{0}\right)\right\}^{-1}<+\infty, \quad n \geq 1,
$$

et en particulier, pour $n=1$, on a le lemme suivant.

Lemma 3. On a presquesûrement

$$
\begin{aligned}
\tilde{E} V & =\lim _{R \rightarrow+\infty}\left(1 / N_{R}\right) \sum_{i=1}^{N_{R}} V\left(D_{i}\right)=\lim _{R \rightarrow+\infty}\left(\frac{V(R)}{N_{R}}\right)=\left\{E\left(\frac{1}{V_{0}}\right)\right\}^{-1} \\
& =\left\{\pi^{1 / 2} \Gamma(d+1) / \Gamma\left(\frac{d+1}{2}\right)\right\} \times\left\{2 \Gamma\left(\frac{d+1}{2}\right) / 2 \pi \Gamma\left(\frac{d}{2}\right)\right\}^{d}
\end{aligned}
$$

soit en dimension $d=2$,

$$
\tilde{\mathrm{E}} \mathrm{V}=1 / \pi .
$$

Généralement, les lois empiriques ne sont pas connues (on ignore notamment les lois des caractéristiques géométriques élémentaires $V, L, S$ ). Cela étant, on doit à Miles, l'expression explicite de la loi du rayon empirique des disque inscrits dans les domaines de la mosaïque, soit le lemme suivant.

Lemme 4. Le rayon empirique $R$ du disque inscrit a pour loi

$$
\tilde{\mathrm{P}}\{\mathrm{R} \geq \mathrm{t}\}=\mathrm{P}\left\{\mathrm{D}_{0} \supset \mathrm{B}(\mathrm{t})\right\}=\exp \{-2 \pi \mathrm{t}\}, \quad \mathrm{t} \geq 0 .
$$

D. Le polyèdre convexe empirique D̃. Dans [18], Miles a indiqué plusieurs constructions possibles d'un polygone convexe aleatoire $\tilde{D} \subset R^{2}$ dont les lois des caractéristiques géométriques associées coïncident avec les lois empiriques définies au point $\mathrm{C}_{\dot{\sim}}$. Ainsi, en particulier, on peut construire un polygone aléatoire convexe $\tilde{D}$ en partant de la loi empirique du rayon du disque inscrit dans les domaines de la mosaïque.

LEMmE 5. On obtient le polygone empirique D̃ de la manière suivante:

(i) On consi dère un cercle de rayon aléatoire $\mathrm{R} \geq 0$ de loi

$$
P\{R \geq t\}=\exp (-2 \pi t), \quad t \geq 0 .
$$

(ii) On construit un triangle $T$ admettant le disque $B(R)$ pour cercle inscrit. Les points de contact des trois côtés du triangle avec le bord du disque étant repérés par des angles aléatoires $\left(\Theta_{1}, \Theta_{2}, \Theta_{3}\right) \in\left[0,2 \pi\left[{ }^{3}\right.\right.$ admettant une Ioi absol ument continue de densité

$$
\frac{2}{3 \pi^{2}}\left|\sin \frac{\theta_{1}-\theta_{2}}{2} \sin \frac{\theta_{2}-\theta_{3}}{2} \sin \frac{\theta_{3}-\theta_{1}}{2}\right|
$$

le vecteur $\left(\Theta_{1}, \Theta_{2}, \Theta_{3}\right)$ étant indépendant du rayon al éatoire $R$.

(iii) Conditionnellement à $R=r$ et à $\left(\Theta_{1}, \Theta_{2}, \Theta_{3}\right)=\left(\theta_{1}, \theta_{2}, \theta_{3}\right)$, on construit le polygone empirique $\tilde{D}$ en prenant un processus poissonien de droites 
$H_{r}$ associés à une mesure de Poisson al éatoire $P_{r}$ de mesure d'intensité

$$
\mu_{\mathrm{r}}(\mathrm{A})=\int_{0}^{2 \pi} \int_{\mathrm{r}}^{+\infty} 1_{\mathrm{A}}(\sigma, \rho) \mathrm{d} \sigma \mathrm{d} \rho, \quad \mathrm{A} \in \mathrm{B}\left(\mathrm{R}^{2}\right),
$$

puis en considérant le polygone convexe, contenant le disque de rayon $r>0$ et obtenu en prenant l'intersection des droites de $H_{r}$ avec les côtés du triangle défini en (ii).

\section{Comportement asymptotique des lois du volume empirique $\mathbf{V}$ et} du volume $\mathbf{V}_{\mathbf{0}}$ du polygone $\mathbf{D}_{\mathbf{0}}$. Nous allons établir le résultat fondamental suivant.

THÉORÈME 1. La relation asymptotique suivante est satisfaite,

$$
\lim _{t \rightarrow+\infty} t^{-1 / 2} \log P\left\{V_{0} \geq t\right\}=\lim _{t \rightarrow+\infty} t^{-1 / 2} \log \tilde{P}\{V \geq t\}=-2 \sqrt{\pi}
$$

Preuve. Nous allons exploiter la construction explicite, fournie par le Lemme 5, du polygone empirique à partir du disque inscrit dans celui-ci. Commençons par remarquer le lemme.

Lemme 6. Désignons par $T_{1, r}, T_{2, r}$ et $T_{3, r}$ les trois composantes connexes de $T \backslash B(r)$ puis par $D_{i, r}=T_{i, r} \cap D, i=1,2,3$, les trois composantes connexes de $\tilde{D} \backslash B(r)$ associées. Alors, conditionnellement à $\left(\Theta_{1}, \Theta_{2}, \Theta_{3}\right)=$ $\left(\theta_{1}, \theta_{2}, \theta_{3}\right)$ et $R=r$, les domaines $D_{i, r}, i=1,2,3$, sont indépendants.

Preuve. Il est clair que, compte-tenu de la définition de la mesure $\mu_{r}$, aucune droite de $H_{r}$ ne coupe le disque centré à l'origine et de rayon $r$. De plus aucune des droites de $H_{r}$ ne peut couper à la fois deux de ces composantes connexes. Ainsi, si $H_{r, 1}, H_{r, 2}$ et $H_{r, 3}$ désignent les droites coupant respectivement $T_{1, r}, T_{2, r}$ et $T_{3, r}$ alors les points de la mesure aléatoire $P_{r}$ correspondant à ces trois ensembles de droites appartiennent à des sousensembles du plan disjoints. II en résulte en particulier que les trois composantes connexes $D_{i, r}, i=1,2,3$, sont indépendantes.

Fixons $\theta_{1}, \theta_{2}, \theta_{3}$ puis, pour tout $r>0$, désignons par $V_{i, r}$ l'aire de la composante connexe $D_{i, r}, i=1,2$, 3 . On a alors le théoreme.

THÉORÈME 2. Pour tout $\varepsilon>0$, il existe une constante $0<\mathrm{A}(\varepsilon)<+\infty$ telle quel'on ait

$$
E \exp \left(\frac{(1-\varepsilon) V_{i, r}}{r}\right) \leq \exp \left(A(\varepsilon) r^{2 / 3}\right), \quad i=1,2,3
$$

pour tout $r>0$. 
Preuve. Pour cela, nous allons majorer les espérances,

$$
\begin{aligned}
E\left\{V_{i, r}^{n}\right\}=\int_{\left\{x_{1}, \ldots, x_{n} \in T_{i, r}\right\}} P\left\{x_{1} \in D_{i, r}, \ldots, x_{n} \in D_{i, r}\right\} d x_{1} \cdots & d x_{n}, \\
& i=1,2,3 .
\end{aligned}
$$

II suffit de se limiter, bien évidemment, au cas $\mathrm{i}=1$.

Repérons un point $x \in T_{1, r} \backslash B(r)$ par

(i) L'angle $\phi$ que fait le segment reliant l'origine [c'est-à-dire, le centre du disque $B(r)$ inscrit] à l'un des deux points de contact du bord $\partial B(r)$ avec les côtés du triangle commun à $T_{1, r}$, avec le segment Ox reliant I'origine au point $\mathrm{x}$;

(ii) L'angle $\alpha \in[0, \pi / 2$ [ que fait le segment reliant le centre du disque au point de contact $t \in \partial \mathrm{B}(\mathrm{r})$ de la tangente à $\partial \mathrm{B}(\mathrm{r})$ issue du point $\mathrm{x}$ avec le segment $O x$ reliant l'origine au point $x$.

Ainsi, en notant par $\rho=\|x\|$ la distance du point $x$ à l'origine, on a

$$
\cos \alpha=\frac{\mathrm{r}}{\rho} \text { et } \tan \alpha=\frac{\|\mathrm{x}-\mathrm{t}\|}{\mathrm{r}} .
$$

Pour tout choix de points $\mathrm{x}_{1}=\left(\phi_{1}, \alpha_{1}\right), \ldots, \mathrm{x}_{\mathrm{n}}=\left(\phi_{\mathrm{n}}, \alpha_{\mathrm{n}}\right) \in \mathrm{T}_{1, \mathrm{r}}$, notons

$$
\begin{aligned}
& \mathrm{P}\left(\left(\phi_{1}, \alpha_{1}\right), \ldots,\left(\phi_{\mathrm{n}}, \alpha_{\mathrm{n}}\right)\right) \\
& \quad=\mathrm{P}\left\{\left(\phi_{1}, \alpha_{1}\right) \in \mathrm{D}_{1, \mathrm{r}}, \ldots,\left(\phi_{\mathrm{n}}, \alpha_{\mathrm{n}}\right) \in \mathrm{D}_{1, \mathrm{r}}\right\}, \quad \mathrm{d} \bar{\alpha}=\mathrm{d} \alpha_{1} \cdots \mathrm{d} \alpha_{\mathrm{n}} .
\end{aligned}
$$

Désignons par $\beta_{\mathrm{i}}$ l'angle au sommet de $\mathrm{T}_{\mathrm{i}, \mathrm{r}}$. On obtient alors

$$
\begin{aligned}
\mathrm{E}\left\{\mathrm{V}_{1, \mathrm{r}}^{\mathrm{n}}\right\}=\left(\mathrm{r}^{2 \mathrm{n}}\right) \mathrm{n} ! \int_{\left\{0<\alpha_{1}<\cdots<\alpha_{\mathrm{n}}<\left(\pi-\beta_{1}\right) / 2\right\}} & \prod_{\mathrm{i}=1}^{\mathrm{n}} \frac{\left(\pi-\beta_{\mathrm{i}}-2 \alpha_{\mathrm{i}}\right) \sin \alpha_{\mathrm{i}}}{\left(\cos \alpha_{\mathrm{i}}\right)^{3}} \\
& \times \mathrm{p}\left(\left(\phi_{1}, \alpha_{1}\right), \ldots,\left(\phi_{\mathrm{n}}, \alpha_{\mathrm{n}}\right)\right) \mathrm{d} \bar{\alpha}
\end{aligned}
$$

Les probabilités $\mathrm{p}\left(\left(\phi_{1}, \alpha_{1}\right), \ldots,\left(\phi_{\mathrm{n}}, \alpha_{\mathrm{n}}\right)\right)$ sont difficiles à évaluer. Commençons par remarquer, dans un premier temps, le lemme.

LEMme 7. On a la majoration

$$
\mathrm{p}\left(\left(\phi_{1}, \alpha_{1}\right), \ldots,\left(\phi_{\mathrm{n}}, \alpha_{\mathrm{n}}\right)\right) \leq \exp \left\{-2 \mathrm{r}\left(\tan \alpha_{\mathrm{n}}-\alpha_{\mathrm{n}}\right)\right\} \text {. }
$$

Preuve. En effet, considérons un point fixé $x \notin B(r)$. En vertu de la construction du processus poissonien de droites $H_{r}$, on a

$$
\mathrm{P}\{\mathrm{x} \in \tilde{\mathrm{D}}\}=\exp \{-[\mathbf{p}(\mathrm{c}(\mathrm{B}(\mathrm{r}) \cup\{\mathrm{x}\}))-2 \pi \mathrm{r}]\},
$$

où, pour tout ensemble $A \subset R^{2}, C(A)$ désigne l'enveloppe convexe de $A$ et $\mathbf{p}(c(A))$ le périmètre de celle-ci. II en résulte trivialement, avec (19), que pour tout point $\mathrm{x}=(\phi, \alpha)$, on a

$$
\mathbf{p}(\mathrm{C}(\mathrm{B}(\mathrm{r}) \cup\{\mathrm{x}\}))-2 \pi \mathrm{r}=2 \mathrm{r}(\tan \alpha-\alpha) \text {. }
$$

Finalement, comme $\mathrm{p}\left(\left(\phi_{1}, \alpha_{1}\right), \ldots,\left(\phi_{\mathrm{n}}, \alpha_{\mathrm{n}}\right)\right) \leq \mathrm{P}\left\{\left(\phi_{\mathrm{n}}, \alpha_{\mathrm{n}}\right) \in \mathrm{D}_{1, \mathrm{r}}\right\}$, on en dé duit le Lemme 7. 
Fixons $\beta$ vérifiant $0<\beta<\pi / 2$ et notons

$$
\begin{aligned}
\mathrm{I}_{\mathrm{n}}(\mathrm{r}, \beta)=\left(\mathrm{r}^{\mathrm{n}}\right) \mathrm{n} ! \int_{\left\{0<\alpha_{1}<\cdots<\alpha_{\mathrm{n}}<\pi / 2, \beta<\alpha_{\mathrm{n}}\right\}} & \prod_{\mathrm{i}=1}^{\mathrm{n}} \frac{\left(\pi-2 \alpha_{\mathrm{i}}\right) \sin \alpha_{\mathrm{i}}}{\left(\cos \alpha_{\mathrm{i}}\right)^{3}} \\
& \times \mathrm{p}\left(\left(\phi_{1}, \alpha_{1}\right), \ldots,\left(\phi_{\mathrm{n}}, \alpha_{\mathrm{n}}\right)\right) \mathrm{d} \bar{\alpha} .
\end{aligned}
$$

Le Lemme 7 nous permet alors d'obtenir le résultat intermédiaire suivant.

Lemme 8. Fixons $0<\varepsilon<1$. II existe $0<\beta(\varepsilon)<\pi / 2$ vérifiant

$$
\lim _{r \rightarrow+\infty} \sum_{n=1}^{+\infty} \frac{(1-\varepsilon)^{n} I_{n}(r, \beta(\varepsilon))}{n !}=0 .
$$

Preuve. La majoration (22) nous permet d'écrire

$$
\begin{aligned}
& \mathrm{I}_{\mathrm{n}}(\mathrm{r}, \beta) \leq\left(\mathrm{r}^{\mathrm{n}}\right) \mathrm{n} ! \int_{\left\{0<\alpha_{1}<\cdots<\alpha_{\mathrm{n}}<\pi / 2, \beta<\alpha_{\mathrm{n}}\right\}} \prod_{\mathrm{i}=1}^{\mathrm{n}} \frac{\left(\pi-2 \alpha_{\mathrm{i}}\right) \sin \alpha_{\mathrm{i}}}{\left(\cos \alpha_{\mathrm{i}}\right)^{3}} \\
& \times \exp \left\{-2 r\left(\tan \alpha_{\mathrm{n}}-\alpha_{\mathrm{n}}\right)\right\} \mathrm{d} \bar{\alpha} \\
& =\mathrm{n}\left(\mathrm{r}^{\mathrm{n}}\right) \int_{\beta}^{\pi / 2}\left\{\int_{0}^{\alpha} \frac{(\pi-2 \mathrm{x}) \sin \mathrm{x}}{(\cos \mathrm{x})^{3}} \mathrm{dx}\right\}^{\mathrm{n}-1} \frac{(\pi-2 \alpha) \sin \alpha}{(\cos \alpha)^{3}} \\
& \times \exp \{-2 r(\tan \alpha-\alpha)\} \mathrm{d} \alpha \\
& =2\left(r^{n+1}\right) \int_{\beta}^{\pi / 2}\left\{\int_{0}^{\alpha} \frac{(\pi-2 x) \sin x}{(\cos x)^{3}} d x\right\}^{n}\left(\frac{1}{\cos ^{2} \alpha}-1\right) \\
& \times \exp \{-2 r(\tan \alpha-\alpha)\} \mathrm{d} \alpha \\
& =2\left(r^{n+1}\right) \int_{\beta}^{\pi / 2}\left[\frac{(\pi-2 \alpha)}{2(\cos \alpha)^{2}}-\frac{\pi}{2}+\tan \alpha\right]^{\mathrm{n}}\left(\frac{1}{\cos ^{2} \alpha}-1\right) \\
& \times \exp \{-2 \mathrm{r}(\tan \alpha-\alpha)\} \mathrm{d} \alpha \text {. }
\end{aligned}
$$

On en déduit la majoration

$$
\begin{aligned}
& \sum_{n=1}^{+\infty} \frac{(1-\varepsilon)^{n} I_{n}(r, \beta)}{n !} \\
&(28) \leq 2 r \int_{\beta}^{\pi / 2}\left(\frac{1}{\cos ^{2} \alpha}-1\right) \times \exp \left\{-r\left[2(\tan \alpha-\alpha)-\frac{(1-\varepsilon)(\pi-2 \alpha)}{2(\cos \alpha)^{2}}\right.\right. \\
&\left.\left.+\frac{(1-\varepsilon) \pi}{2}-(1-\varepsilon) \tan \alpha\right]\right\} \mathrm{d} \alpha
\end{aligned}
$$


Le Lemme 8 en découle car, comme il est facile de voir, il existe $\beta(\varepsilon)<\pi / 2$ tel que I'on ait

$$
2(\tan \alpha-\alpha)-\frac{(1-\varepsilon)(\pi-2 \alpha)}{2(\cos \alpha)^{2}}+\frac{(1-\varepsilon) \pi}{2}-(1-\varepsilon) \tan \alpha>0
$$

pour tout $\alpha$, vérifiant $\beta(\varepsilon) \leq \alpha<\pi / 2$.

Le Lemme 7 nous fournit une estimation "suffisamment" précise lorsque l'un des points $x_{1}, \ldots, x_{n}$ intervenant dans la probabilité (20) est situé à une distance du centre du disque inscrit supérieure à $r / \cos \beta(\varepsilon)$. II reste à évaluer cette probabilité de manière plus fine lorsque tous les points $x_{1}, \ldots, x_{n}$ sont pris dans le domaine

$$
\mathrm{C}_{\mathrm{r}}(\varepsilon)=\left\{\mathrm{x} \in \Delta(\mathrm{r}) ; \mathrm{r} \leq\|\mathrm{x}\| \leq \frac{\mathrm{r}}{\cos \beta(\varepsilon)}\right\}
$$

où

$$
\Delta(r)=\left([-r, r] \times R^{+}\right) \backslash B(r) \subset R^{2}
$$

est la partie de la bande $[-r, r] \times \mathrm{R}^{+}$du plan située "au dessus" du disque inscrit $B(r)$. C'est la partie délicate de la démonstration.

Commençons par établir un résultat de nature isopérimétrique. Pour toute courbe rectifiable $C$ on désignera par $\bar{p}(C)$ sa longueur.

THÉORÈmE 3. Soit $C$ une courbe convexe située dans $\Delta$ (1) et admettant pour extrêmités les points $a=(-1,0)$ et $b=(0,1)$ [ c'est-à-dire, les points de contact du cercle $\mathrm{C}_{0}=\partial \mathrm{B}(1)$ avec les bords de la bande]. Désignons par $\mathrm{A}(\mathrm{C})$ le domaine de $\Delta(1)$ compris entre $C$ et le cercle $C_{0}$ par $\partial A(C)$ sa frontière, et par $\partial_{0} A(C)=\partial A(C) \cap C_{0}$ la partie de $C$ située sur le cercle $C_{0}$. Soit finale ment $V_{c}$ I'aire du domaine $A(C)$. La majoration suivante est al ors satisfaite,

$$
\overline{\mathrm{p}}\left(\mathrm{C} \backslash \partial_{0} \mathrm{~A}(\mathrm{C})\right)-\overline{\mathrm{p}}\left(\partial_{0} \mathrm{~A}(\mathrm{C})\right)-\mathrm{V}_{\mathrm{c}} \geq 0,
$$

l'égalité n'ayant lieu que pour les courbes $C_{h}, h>0$, obtenues en prenant la réunion des segments $\{-1\} \times[0, h]$ et $\{1\} \times[0, h]$ et du demi-cercle de rayon un et d'extrêmités $(-1, h)$ et $(1, h)$.

Preuve. On vérifie, tout d'abord, trivialement, par un calcul direct, que pour les courbes $C_{h}, h>0$, on a

$$
\overline{\mathrm{p}}\left(\mathrm{C} \backslash \partial_{0} \mathrm{~A}\left(\mathrm{C}_{\mathrm{h}}\right)\right)-\overline{\mathrm{p}}\left(\partial_{0} \mathrm{~A}\left(\mathrm{C}_{\mathrm{h}}\right)\right)-\mathrm{V}_{\mathrm{c}}=0 .
$$

Par ailleurs, considérons le domaine U(C) admettant pour frontière la réunion de la courbe $C$ et du segment $[-1,1] \times\{0\}$ et soit $I>\pi$ un nombre fixé. II est facile de voir que le problème posé consiste à trouver la courbe convexe $C$, de longueur I, pour laquelle l'aire du domaine U(C) est maximale. En appliquant le procédé de symétrisation de Steiner (voir [21], par exemple) par 
rapport à l'axe $\{0\} \times R^{+}$, on se ramène au cas où la courbe $C$ est symétrique par rapport à cet axe. On peut alors partitionner la courbe $C$ sous la forme

$$
\mathrm{C}=\mathrm{C}_{1} \cup \mathrm{C}_{2} \cup \mathrm{C}_{3}
$$

de sorte que:

(i) Les courbes $C_{1}$ et $C_{2}$ soient issues respectivement des points a et b;

(ii) Les courbes $C_{1}$ et $C_{2}$ soient symétriques par rapport à l'axe $\{0\} \times R^{+}$;

(iii) On a $\overline{\mathrm{p}}\left(\mathrm{C}_{1}\right)=\overline{\mathrm{p}}\left(\mathrm{C}_{2}\right)=(\mathrm{I}-\pi) / 2$.

Le domaine $\mathrm{U}(\mathrm{C})$ se partitionne, à son tour, sous la forme

$$
U(C)=U_{1} \cup U_{2}
$$

où

(a) La frontière du domaine convexe $\mathrm{U}_{1}$ est constituée des courbes $\mathrm{C}_{1}$ et $C_{2}$, du segment $a b$ et du segment de droite $a^{\prime} b^{\prime}$ reliant les deux extrêmités respectives $a^{\prime}$ et $b^{\prime}$ de $C_{1}$ et $C_{2}$ différentes des points $a$ et $b$;

(b) La frontière du domaine convexe $U_{2}$ est constituée de la courbe $C_{3}$ et du segment $a^{\prime} b^{\prime}$.

En vertu du théorème isopérimétrique classique, l'aire $\mathrm{V}\left(\mathrm{U}_{2}\right)$ du domaine $\mathrm{U}_{2}$ vérifie la majoration

$$
\mathrm{V}\left(\mathrm{U}_{2}\right) \geq \pi / 2,
$$

l'égalité n'étant obtenue que si $V\left(U_{2}\right)$ est un demicercle de rayon égal à un.

Considérons, par ailleurs, le domaine rectangulaire $\mathrm{R}$ inscrit dans la bande $\Delta(1)$, admettant le segment ab pour base et pour hauteur

$$
\mathrm{h}=\frac{\mathrm{l}-\pi}{2} \text {. }
$$

Les courbes $C_{1}$ et $C_{2}$ étant convexes le domaine $R$ contient le domaine $U_{1}$ et donc son aire $V(R)$ vérifie trivialement la majoration

$$
\mathrm{V}(\mathrm{R}) \geq \mathrm{V}\left(\mathrm{U}_{1}\right) \text {. }
$$

Considérons finalement le domaine convexe $U_{m}$ admettant pour frontière la courbe obtenue en prenant la réunion du segment $a b$, des deux segments de droites $\{-1\} \times[0, \mathrm{~h}]$ et $\{1\} \times[0, \mathrm{~h}]$ et du demicercle de rayon un et d'extrêmités $(-1, h)$ et $(1, h)$. II résulte, de ce qui précède, que l'on a

$$
\overline{\mathrm{p}}\left(\partial \mathrm{U}_{\mathrm{m}}\right)=\overline{\mathrm{p}}(\partial \mathrm{U}(\mathrm{C}))
$$

l'égalité n'ayant lieu que si la courbe $C_{3}$ est un demi-cercle de rayon égal à un.

Ainsi le domaine $U_{m}$ est la solution du problème considéré et tout résulte alors de (33).

Le Théorème 3 va nous permettre d'obtenir une estimation de la probabilité $P\left\{x_{i} \in D_{1, r} \cap C_{r(\varepsilon)}, i=1, \ldots, n\right\}$ plus précise que celle fournie par le Lemme 7. 
Notons

$$
\begin{aligned}
J_{n}(r, \varepsilon)=\int_{\left\{x_{i} \in T_{1, r}, x_{i} \in C_{r}(\varepsilon), i=1, \ldots, n\right\}} & P\left\{x_{1} \in D_{1, r}, \ldots, x_{n} \in D_{1, r}\right\} \\
& \times d x_{1} \cdots d_{n} .
\end{aligned}
$$

Lemme 9. II existe une constante $0<\mathrm{C}^{\prime}(\varepsilon)<+\infty$, vérifiant

$$
\sum_{n=1}^{+\infty} \frac{J_{n}(r, \varepsilon)}{r^{n} n !} \leq \exp \left(c^{\prime}(\varepsilon) r^{2 / 3}\right) \text { pour tout } r>0 \text {. }
$$

Preuve. Pour tout $n \geq 1$, désignons par $H_{n}$ l'ensemble des points $\left(\mathrm{x}_{1}, \ldots, \mathrm{x}_{\mathrm{n}}\right) \in\left[\mathrm{C}_{1}(\varepsilon)\right]^{\mathrm{n}}$ vérifiant la propriété $(\mathrm{P} 1)$ suivante.

(P1) Pour tout $i=1, \ldots, n$, le point $x_{i}$ n'appartient pas à l'enveloppe convexe fermée $c\left(\left\{x_{j}, j=1, \ldots, n, j \neq i\right\} \cup B(1)\right)$ des points $x_{j}, j=1, \ldots, n$, $j \neq i$, et du disque $B(1)$.

Lorsque le vecteur $\left(x_{1}, \ldots, x_{n}\right)$ appartient à $H_{n}$, la frontière de l'enveloppe convexe $c\left(\left\{x_{j}, j=1, \ldots, n\right\} \cup B(1)\right)$ est de la forme

$$
\partial C\left(\left\{x_{j}, j=1, \ldots, n\right\} \cup B(1)\right)=L_{1} \cup L_{2},
$$

où

(i) $\mathrm{L}_{1}$ est une ligne polygonale tangente au cercle $\mathrm{C}=\partial \mathrm{B}$ (1) et admettant les points $x_{j}, j=1, \ldots, n$, comme points extrêmaux;

(ii) $L_{2}$ et un arc du cercle $C$.

Désignons par

$$
A\left(x_{1}, \ldots, x_{n}\right)=\left[C\left(\left\{x_{j}, j=1, \ldots, n\right\} \cup B(1)\right)\right] \backslash B(1)
$$

le domaine borné de $\Delta(1)$ admettant la courbe polygonale $L_{1}$ pour frontière et notons

$$
\bar{p}_{0}\left(x_{1}, \ldots, x_{n}\right)=\bar{p}\left(c\left(B(1) \cup\left\{x_{i}, i=1, \ldots, n\right\}\right)\right)-2 \pi .
$$

Notons I'inégalité suivante:

$$
\begin{aligned}
& \int_{\left\{x_{i} \in D_{1,1} \cap C_{1}(\varepsilon), i=1, \ldots, n\right\}} P\left\{x_{1} \in D_{1,1}, \ldots, x_{n} \in D_{1,1}\right\} d x_{1} \cdots d x_{n} \\
& \leq \int_{\left\{x_{i} \in C_{1}(\varepsilon), i=1, \ldots, n\right\}} \exp \left\{-\bar{p}_{0}\left(x_{1}, \ldots, x_{n}\right)\right\} d x_{1} \cdots d x_{n} \\
& =\sum_{p=1}^{n-1} \frac{n !}{(n-p) ! p !} \int_{\left\{\left(x_{1}, \ldots, x_{p}\right) \in H_{p}\right\}}\left[\int_{\left\{x_{p+1}, \ldots, x_{n} \in A\left(x_{1}, \ldots, x_{p}\right)\right\}} d x_{p+1} \cdots d x_{n}\right] \\
& \quad \times \exp \left\{-\bar{p}_{0}\left(x_{1}, \ldots, x_{p}\right)\right\} d x_{1} \cdots d x_{p} \\
& \text { (46) } \quad+\int_{\left\{\left(x_{1}, \ldots, x_{n}\right) \in H_{n}\right\}} \exp \left\{-\bar{p}_{0}\left(x_{1}, \ldots, x_{n}\right)\right\} d x_{1} \cdots d x_{n}
\end{aligned}
$$




$$
\begin{aligned}
& =\sum_{p=1}^{n-1} \frac{n !}{(n-p) ! p !} \int_{\left\{\left(x_{1}, \ldots, x_{p}\right) \in H_{p}\right\}}\left[\int_{A\left(x_{1}, \ldots, x_{p}\right)} d x\right]^{n-p} \\
& \quad \times \exp \left\{-\bar{p}_{0}\left(x_{1}, \ldots, x_{p}\right)\right\} d x_{1} \cdots d x_{p} \\
& \quad+\int_{\left\{\left(x_{1}, \ldots, x_{n}\right) \in H_{n}\right\}} \exp \left\{-\bar{p}_{0}\left(x_{1}, \ldots, x_{n}\right)\right\} d x_{1} \cdots d x_{n}
\end{aligned}
$$

puis appliquons l'inégalité isopérimétrique (32). On obtient

$$
\begin{gathered}
\int_{\left\{x_{i} \in C_{1}(\varepsilon), i=1, \ldots, n\right\}} P\left\{x_{1} \in D_{1,1}, \ldots, x_{n} \in D_{1,1}\right\} d x_{1} \cdots d x_{n} \\
\leq \sum_{p=1}^{n-1} \frac{n !}{(n-p) ! p !} \int_{\left\{\left(x_{1}, \ldots, x_{p}\right) \in H_{p}\right\}}\left[\bar{p}_{0}\left(x_{1}, \ldots, x_{p}\right]^{n-p}\right. \\
\quad \times \exp \left\{-\bar{p}_{0}\left(x_{1}, \ldots, x_{p}\right)\right\} d x_{1} \cdots d x_{p} \\
+\int_{\left\{\left(x_{1}, \ldots, x_{n}\right) \in H_{n}\right\}} \exp \left\{-\bar{p}_{0}\left(x_{1}, \ldots, x_{n}\right)\right\} d x_{1} \cdots d x_{n} .
\end{gathered}
$$

On en déduit la majoration

$$
\begin{aligned}
& \sum_{n=1}^{+\infty} \frac{J_{n}(1, \varepsilon)}{n !} \\
& \leq \sum_{p=1}^{+\infty}\left\{\sum_{n=p}^{+\infty} \frac{1}{(n-p) ! p !}\right. \\
& \quad \times \int_{\left\{\left(x_{1}, \ldots, x_{p}\right) \in H_{p}\right\}}\left[\bar{p}_{0}\left(x_{1}, \ldots, x_{p}\right)\right]^{n-p} \\
& \left.\quad \times \exp \left\{-\bar{p}_{0}\left(x_{1}, \ldots, x_{p}\right)\right\} d x_{1} \cdots d x_{p}\right\} \\
& =\sum_{p=1}^{+\infty} \frac{1}{p !} \int_{\left\{\left(x_{1}, \ldots, x_{p}\right) \in H_{p}\right\}} d x_{1} \cdots d x_{p} .
\end{aligned}
$$

Fixons $\left(x_{1}, \ldots, x_{p}\right) \in H_{p}$. Les points $x_{1}, \ldots, x_{p}$ appartiennent tous à la même courbe polygonale $L_{1}$ [voir (43)] de périmètre

$$
\overline{\mathrm{p}}\left(\mathrm{L}_{1}\right) \leq \tan \beta(\varepsilon)+\frac{\beta(\varepsilon)}{\cos \beta(\varepsilon)}=\mathrm{I}(\varepsilon) .
$$

II en résulte que pour tout $A>0$ on $a$

(50) $\quad \operatorname{card}\left\{\mathrm{i} \in(1, \ldots, \mathrm{p}-1) ;\left\|\mathrm{x}_{\mathrm{i}}-\mathrm{x}_{\mathrm{i}+1}\right\| \geq \frac{\mathrm{I}(\varepsilon)}{\mathrm{nA}}\right\} \leq \operatorname{ent}(\mathrm{nA})$ 
où ent $(x)$ désigne la partie entière de $x$. On en déduit facilement qu'il existe une constante $\mathrm{c}(\varepsilon)>0$ vérifiant

$$
\int_{\left\{\left(\mathrm{x}_{1}, \ldots, \mathrm{x}_{\mathrm{p}}\right) \in \mathrm{H}_{\mathrm{p}}\right\}} \mathrm{dx}_{1} \cdots \mathrm{dx_{ \textrm {p } }} \leq\left[\frac{\mathrm{c}(\varepsilon)}{\mathrm{p}}\right]^{\mathrm{p} / 2}, \quad \mathrm{p} \geq 1,
$$

d'où la majoration

$$
\sum_{n=1}^{+\infty} \frac{J_{n}(1, \varepsilon)}{n !} \leq \sum_{p=1}^{+\infty}\left(\frac{1}{p !}\right)\left[\frac{c(\varepsilon)}{p}\right]^{p / 2} .
$$

Par un argument immédiat d'homogénéité on en déduit que

$$
\sum_{n=1}^{+\infty} \frac{J_{n}(r, \varepsilon)}{r^{n} n !} \leq \sum_{p=1}^{+\infty} \frac{r^{p}}{p !}\left[\frac{c(\varepsilon)}{p}\right]^{p / 2} \leq \exp \left(c^{\prime}(\varepsilon) r^{2 / 3}\right), \quad r>0,
$$

où $C^{\prime}(\varepsilon)>0$ est une constante, ce qui termine la preuve du Lemme 9 .

Fin de la preuve du Théorème 2. Elle découle des Lemmes 8 et 9 . En effet on a

$$
\mathrm{E}\left\{\mathrm{V}_{1, \mathrm{r}}^{\mathrm{n}}\right\}=\mathrm{r}^{\mathrm{n}} \mathrm{I}_{\mathrm{n}}(\mathrm{r}, \beta(\varepsilon))+\mathrm{J}_{\mathrm{n}}(\mathrm{r}, \varepsilon)
$$

et donc d'après (26) et (41) on a

$$
E\left\{\exp \left(\frac{(1-\varepsilon) V_{i, r}}{r}\right)\right\} \leq \exp \left(c^{\prime \prime}(\varepsilon) r^{2 / 3}\right) \text { pour tout } r>0,
$$

où $C^{\prime \prime}(\varepsilon)$ est une constante. C'est le résultat souhaité.

FIN DE LA PREUVE dU ThÉORÈme 1. Nous sommes maintenant en mesure d'établir le Théorème 1 . Nous déduisons, tout d'abord, de (17) et du Lemme 6 que pour tout $\mathrm{t}>0$ on a

$$
\begin{aligned}
\mathrm{P}\left\{\mathrm{V}_{\mathrm{r}} \geq \mathrm{t}-\pi \mathrm{r}^{2}\right\} & \leq \exp \left\{-\frac{(1-\varepsilon)\left(\mathrm{t}-\pi \mathrm{r}^{2}\right)}{\mathrm{r}}+\mathrm{C}^{\prime \prime}(\varepsilon) \mathrm{r}^{2 / 3}\right\} \\
& \leq \mathrm{A}(\varepsilon) \exp \left\{-\frac{(1-\varepsilon)\left(\mathrm{t}-\pi \mathrm{r}^{2}\right)}{\mathrm{r}}+\varepsilon \pi \mathrm{r}\right\}, \quad \mathrm{r}>0,
\end{aligned}
$$

où $\mathrm{A}(\varepsilon)>0$ est une constante.

Par ailleurs $t>0$ étant fixé, on a

$$
\tilde{\mathrm{P}}\{\mathrm{V} \geq \mathrm{t} \mid \mathrm{R}=\mathrm{r}\}= \begin{cases}1, & \text { si } \pi \mathrm{r}^{2} \geq \mathrm{t}, \\ \mathrm{P}\left\{\mathrm{V}_{\mathrm{r}} \geq \mathrm{t}-\pi \mathrm{r}^{2}\right\}, & \text { si } \pi \mathrm{r}^{2}<\mathrm{t} .\end{cases}
$$

II en résulte que [avec (12)]

$$
\tilde{\mathrm{P}}\{\mathrm{V} \geq \mathrm{t}\}=\int_{\sqrt{\mathrm{t} / \pi}}^{+\infty} \exp \{-2 \pi \mathrm{r}\} 2 \pi \mathrm{dr}+\mathrm{K}(\mathrm{t})
$$


où

$$
\begin{aligned}
\mathrm{K}(\mathrm{t}) & =\int_{0}^{(\mathrm{t} / \pi)^{1 / 2}} \exp \{-2 \pi \mathrm{r}\} \tilde{\mathrm{P}}\{\mathrm{V} \geq \mathrm{t} \mid \mathrm{R}=\mathrm{r}\} 2 \pi \mathrm{dr} \\
& =\int_{0}^{(\mathrm{t} / \pi)^{1 / 2}} \exp \{-2 \pi \mathrm{r}\} \mathrm{P}\left\{\mathrm{V}_{\mathrm{r}} \geq \mathrm{t}-\pi \mathrm{r}^{2}\right\} 2 \pi \mathrm{dr} \\
& \leq 2 \pi \mathrm{A}(\varepsilon) \int_{0}^{(\mathrm{t} / \pi)^{1 / 2}} \exp \left\{-2 \pi \mathrm{r}-\frac{(1-\varepsilon)\left(\mathrm{t}-\pi \mathrm{r}^{2}\right)}{\mathrm{r}}+\varepsilon \pi \mathrm{r}\right\} \mathrm{dr} \\
& \leq 2 \pi \mathrm{A}(\varepsilon) \int_{0}^{+\infty} \exp \left\{-2 \pi \mathrm{r}-\frac{(1-\varepsilon)\left(\mathrm{t}-\pi \mathrm{r}^{2}\right)}{\mathrm{r}}+\varepsilon \pi \mathrm{r}\right\} \mathrm{dr} \\
& =4 \pi \mathrm{A}(\varepsilon) \sqrt{\frac{\mathrm{t}(1-\varepsilon)}{\pi}} \mathrm{K}_{1}(2 \sqrt{\mathrm{t}(1-\varepsilon) \pi}),
\end{aligned}
$$

où $K_{1}(x)$ et la fonction de Bessel modifiée de troisième espèce.

Par ailleurs on sait que (voir [14])

$$
\mathrm{K}_{1}(\mathrm{x}) \underset{\mathrm{x} \rightarrow+\infty}{\sim} \sqrt{\frac{\pi}{2 \mathrm{x}}} \exp \{-\mathrm{x}\}
$$

Avec (58) on en déduit que

$$
\lim _{\mathrm{t} \rightarrow+\infty} \mathrm{t}^{-1 / 2} \log \tilde{\mathrm{P}}\{\mathrm{V} \geq \mathrm{t}\}=-2 \sqrt{\pi}
$$

c'est-à-dire, l'équivalent, du Théorème 1, relatif à la distribution empirique de l'aire des polygones convexes.

Pour obtenir l'équivalent annoncé, dans le Théorème 1, pour l'aire de la cellule de Crofton, nous allons faire appel à l'identité suivante [voir (7) du Lemme 2]:

$$
\begin{aligned}
\tilde{P}\{\mathrm{~V} \geq t\} & =\lim _{\mathrm{R} \rightarrow+\infty}\left(1 / \mathrm{N}_{\mathrm{R}}\right) \sum_{\mathrm{i}=1}^{\mathrm{N}_{\mathrm{R}}} 1_{[\mathrm{t},+\infty]}\left(\mathrm{X}\left(\mathrm{D}_{\mathrm{i}}\right)\right) \\
& =(1 / \pi)\left\{\mathrm{E}\left(1_{[\mathrm{t},+\infty]}\left(\mathrm{V}_{0}\right)\right) / \mathrm{V}_{0}\right\},
\end{aligned}
$$

reliant la distribution du volume empirique $\mathrm{V}$ à la distribution du volume $\mathrm{V}_{0}$ de la cellule de Crofton $D_{0}$. On voit donc, avec (61) et (62), que pour tout $\varepsilon>0$, fixé, il existe une constante $\mathrm{A}^{\prime}(\varepsilon)$ vérifiant

$$
\int_{\left\{\mathrm{V}_{0} \geq \mathrm{t}\right\}} \frac{\mathrm{dP}}{\mathrm{V}_{0}} \leq \mathrm{A}^{\prime}(\varepsilon) \exp (-2(1-\varepsilon) \sqrt{\pi \mathrm{t}}) \text { pour tout } \mathrm{t} \geq 0
$$

Rappelons encore la formule (voir [6])

$$
\mathrm{EV}_{0}^{\mathrm{n}} \leq \frac{\mathrm{n} \pi^{\mathrm{n}} \Gamma(2 \mathrm{n})}{2^{2 \mathrm{n}-1}}, \quad \mathrm{n} \geq 1
$$

donnant un majorant des moments d'ordre $\mathrm{n} \geq 1$ du volume $\mathrm{V}_{0}$. II en résulte qu'il existe une constante $0<\mathrm{c}<2$, vérifiant

$$
\mathrm{P}\left\{\mathrm{V}_{0} \geq \mathrm{t}\right\} \leq \exp (-\mathrm{c} \sqrt{\mathrm{t}}), \quad \mathrm{t}>0 \text {. }
$$


Nous en déduisons la majoration

$$
\begin{aligned}
\mathrm{P}\left\{\mathrm{V}_{0} \geq \mathrm{t}\right\} & =\mathrm{P}\left\{\mathrm{V}_{0} \geq\left(\frac{2}{\mathrm{c}}\right)^{2} \pi \mathrm{t}\right\}+\mathrm{P}\left\{\mathrm{t} \leq \mathrm{V}_{0} \leq\left(\frac{2}{\mathrm{c}}\right)^{2} \pi \mathrm{t}\right\} \\
& \leq \exp (-2 \sqrt{\pi \mathrm{t}})+\mathrm{P}\left\{\mathrm{t} \leq \mathrm{V}_{0} \leq\left(\frac{2}{\mathrm{c}}\right)^{2} \pi \mathrm{t}\right\} \\
& \leq \exp (-2 \sqrt{\pi \mathrm{t}})+\left(\frac{2}{\mathrm{c}}\right)^{2} \pi \mathrm{t} \int_{\left\{\mathrm{t} \leq \mathrm{V}_{0}\right\}} \frac{\mathrm{dP}}{\mathrm{V}_{0}}
\end{aligned}
$$

ce qui, avec (62) et (63), fournit l'équivalent souhaité.

3. Comportement asymptotique de la première valeur propre du Laplacien pour la cellule de Crofton ainsi que pour le polygone empirique. L'estimation (fine) de la fonction de répartition du volume empirique, fournie par le Théorème 1 , va nous permettre de préciser le comportement asymptotique de la première valeur propre du Laplacien pour les grands domaines. Dans l'énoncé qui suit on désigne:

1. Par $\mu_{1,0}$ la première valeur propre du Laplacien (pour le problème de Dirichlet) du disque inscrit dans le domaine $D_{0}$

2. Par $\lambda_{1,0}$ la première valeur propre du Laplacien (pour le problème de Dirichlet) du domaine $\mathrm{D}_{0}$

3. Par $\tilde{E}\left[\exp \left(-\mathrm{t} \lambda_{1}\right)\right]=\lim _{\mathrm{R} \rightarrow+\infty}\left(1 / \mathrm{N}_{\mathrm{R}}\right) \sum_{\mathrm{i}=1}^{\mathrm{N}_{\mathrm{R}}} \exp \left(-\mathrm{t} \lambda_{1, \mathrm{i}}\right)$ la transformée de Laplace empirique de la première valeur propre des domaines de la mosaïque (voir [6])

4. Par $\tilde{E}\left[\exp \left(-t \mu_{1}\right)\right]=\lim _{R \rightarrow+\infty}\left(1 / N_{R}\right) \sum_{i=1}^{N_{R}} \exp \left(-t \mu_{1, i}\right)$ la transformée de Laplace empirique de la première valeur propre des disques inscrits dans les domaines de la mosaïque.

THÉORÈME 4. On a

$$
\begin{aligned}
\lim _{\mathrm{R} \rightarrow+\infty} \mathrm{t}^{-1 / 3} \log \tilde{\mathrm{E}}\left[\exp \left(-\mathrm{t} \lambda_{1}\right)\right] & =\lim _{\mathrm{R} \rightarrow+\infty} \mathrm{t}^{-1 / 3} \log \tilde{\mathrm{E}}\left[\exp \left(-\mathrm{t} \mu_{1}\right)\right] \\
& =\lim _{\mathrm{R} \rightarrow+\infty} \mathrm{t}^{-1 / 3} \log \mathrm{E}\left[\exp \left(-\mathrm{t} \mu_{1,0}\right)\right] \\
& =\lim _{\mathrm{R} \rightarrow+\infty} \mathrm{t}^{-1 / 3} \log \mathrm{E}\left[\exp \left(-\mathrm{t} \lambda_{1,0}\right)\right] \\
& =-\left(27 \pi^{2} \mathrm{j}_{0}^{2}\right)^{1 / 3},
\end{aligned}
$$

où $\mathrm{j}_{0} \sim 2,405$ désigne la plus petite racine positive non-nulle de la fonction de Bessel J 0 . 
PReUVe. La première valeur propre d'une boule $B(r)$ est égale à $j_{0}^{2} / r^{2}$. On en déduit, avec (12), que

$$
\begin{aligned}
\tilde{E}\left[\exp \left(-\mathrm{t} \mu_{1}\right)\right] & =\int_{0}^{+\infty} \exp \left(-2 \pi \mathrm{x}-\frac{\mathrm{tj}_{0}^{2}}{\mathrm{x}^{2}}\right) 2 \pi \mathrm{dx} \\
& =2 \pi \mathrm{t}^{1 / 3} \int_{0}^{+\infty} \exp \left[-\mathrm{t}^{1 / 3}\left(2 \pi \mathrm{x}+\frac{\mathrm{tj}_{0}^{2}}{\mathrm{x}^{2}}\right)\right] \mathrm{dx} .
\end{aligned}
$$

La méthode classique de Laplace fournit, alors, l'équival ent, lorsque $t \rightarrow+\infty$, suivant:

$$
\int_{0}^{+\infty} \exp \left[-t^{1 / 3}\left(x+\frac{a}{x^{2}}\right)\right] d x \sim \sqrt{\frac{6 \pi}{(2 a t)^{1 / 3}}} \exp \left\{-\left(\frac{27 a t}{4}\right)^{1 / 3}\right\}
$$

II en résulte immédiatement que

$$
\lim _{\mathrm{t} \rightarrow+\infty} \mathrm{t}^{-1 / 3} \log \tilde{E}\left[\exp \left(-\mathrm{t} \mu_{1}\right)\right]=-\left(27 \pi^{2} \mathrm{j}_{0}^{2}\right)^{1 / 3} .
$$

Par ailleurs, comme la première valeur propre du Laplacien d'un domaine minore la première valeur du Laplacien de tout sous-domaine on a

$$
\liminf _{\mathrm{t} \rightarrow+\infty} \mathrm{t}^{-1 / 3} \log \tilde{E}\left[\mathrm{e}^{-\mathrm{t} \lambda_{1}}\right] \geq \lim _{\mathrm{t} \rightarrow+\infty} \mathrm{t}^{-1 / 3} \log \tilde{E}\left[\exp \left(-\mathrm{t} \mu_{1}\right)\right] .
$$

Pour établir la majoration inverse, nous allons faire appel à l'inégalité de Faber-Krahn (voir [12]),

$$
\lambda_{1} \geq \pi \mathrm{j}_{0}^{2} / \mathrm{V}(\mathrm{D})
$$

satisfaite par la première valeur propre d'un domaine $D$ quelconque.

Fixons $\varepsilon>0$. Il résulte de (72) et du Théorème 1 qu'il existe $A(\varepsilon)>0$ telle que

$$
\begin{aligned}
\tilde{\mathrm{E}}\left[\exp \left(-\mathrm{t} \lambda_{1}\right)\right] & \leq \tilde{\mathrm{E}}\left[\exp \left\{-\mathrm{t} \pi \mathrm{j}_{0}^{2} / \mathrm{V}\right\}\right] \\
& =\int_{0}^{+\infty}\left[\frac{\mathrm{t} \pi \mathrm{j}_{0}^{2}}{\mathrm{x}^{2}}\right] \exp \left(-\frac{\mathrm{t} \pi \mathrm{j}_{0}^{2}}{\mathrm{x}}\right) \tilde{\mathrm{P}}\{\mathrm{V} \geq \mathrm{x}\} \mathrm{dx} \\
& \leq \mathrm{A}(\varepsilon) \int_{0}^{+\infty}\left[\frac{\mathrm{t} \pi \mathrm{j}_{0}^{2}}{\mathrm{x}^{2}}\right] \exp \left\{-2(1-\varepsilon) \sqrt{\pi} \mathrm{x}-\frac{\mathrm{t} \pi \mathrm{j}_{0}^{2}}{\mathrm{x}}\right\} \mathrm{dx} \\
& \leq \mathrm{A}(\varepsilon) \int_{0}^{+\infty}\left[\frac{\mathrm{t}^{2 / 3} \pi \mathrm{j}_{0}^{2}}{\mathrm{x}^{2}}\right] \exp \left\{-\mathrm{t}^{1 / 3}\left[2(1-\varepsilon) \sqrt{\pi} \mathrm{x}+\frac{\pi \mathrm{j}_{0}^{2}}{\mathrm{x}}\right]\right\} \mathrm{dx} .
\end{aligned}
$$

En faisant appel, à nouveau, à la méthode de Laplace nous en déduisons

$$
\operatorname{limsupt}_{\mathrm{t} \rightarrow+\infty} \mathrm{t}^{-1 / 3} \log \tilde{E}\left[\exp \left(-\mathrm{t} \lambda_{1}\right)\right] \leq-\left\{(1-\varepsilon)^{2} 27 \pi^{2} \mathrm{j}_{0}^{2}\right\}^{1 / 3}
$$


Les inégalités (70), (71) et (74) fournissent alors la relation asymptotique

$$
\lim _{t \rightarrow+\infty} t^{-1 / 3} \log \tilde{E}\left[\exp \left(-t \lambda_{1}\right)\right]=-\left(27 \pi^{2} j_{0}^{2}\right)^{1 / 3}
$$

souhaitée.

On démontre de la même manière les deux autres relations asymptotiques du Théorème 4. Pour cela on fera appel à l'estimation (16) satisfaite pour l'aire du domaine $D_{0}$ ainsi qu'à la formule (12) fournissant la loi du rayon de la boule $\mathrm{B}_{0}$ centrée à l'origine et inscrite dans $\mathrm{D}_{0}$.

4. Comportement asymptotique du périmètre $Y_{0}$ de l'enveloppe convexe de la trajectoire du pont brownien plan. Soient $D_{i}, i=$ $1, \ldots, N_{R}$, les domaines polygonaux convexes, associés à la mesure de Poisson aléatoire $P=\left\{x_{n}, n \geq 1\right\}$ définie dans l'espace euclidien $\mathbf{R}^{2}$, inclus dans le disque ouvert $B(R)$. Désignons par

$$
\phi_{\mathrm{i}}(\mathrm{t})=\sum_{\mathrm{n}=1}^{+\infty} \exp \left(-\mathrm{t} \lambda_{\mathrm{n}, \mathrm{i}}\right), \quad \mathrm{t}>0
$$

la fonction spectrale [6], associée aux valeurs propres $\lambda_{\mathrm{n}, \mathrm{i}}, \mathrm{n} \geq 1$, du laplacien $\Delta=\partial^{2} / \partial \mathrm{x}^{2}+\partial^{2} / \partial \mathrm{y}^{2}$, pour le problème de Dirichlet sur le domaine $\mathrm{D}_{\mathrm{i}}$, puis posons

$$
\Phi_{R}(t)=\left(1 / N_{R}\right) \sum_{i=1}^{N_{R}} \phi_{i}(t) .
$$

Considérons, par ailleurs, les ponts browniens,

$$
\left(\mathrm{W}_{0, \mathrm{t}}(\mathrm{s})\right)_{0 \leq \mathrm{s} \leq \mathrm{t}}, \mathrm{W}_{0, \mathrm{t}}(0) \equiv \mathrm{W}_{0, \mathrm{t}}(\mathrm{t}) \equiv 0, \quad \mathrm{t}>0
$$

du plan, démarrant de l'origine; c'est-à-dire, le mouvement brownien plan, démarrant de l'origine et conditionné pour y repasser à l'instant $t>0$. Soient

$$
\mathbf{w}_{0, t}=\left\{\mathrm{w}_{0, \mathrm{t}}(\mathrm{s}), 0 \leq \mathrm{s} \leq \mathrm{t}\right\}, \quad \mathrm{t}>0
$$

les trajectoires associées puis $Y_{0}$ le périmètre de l'enveloppe convexe de $\mathbf{W}_{0,1}$. Dans [6] nous avons démontré que, presque sûrement pour tout $t>0$, on a

$$
\Phi(\mathrm{t})=\lim _{\mathrm{R} \rightarrow+\infty} \Phi_{\mathrm{R}}(\mathrm{t})=\left(1 / 4 \pi^{2} \mathrm{t}\right) \mathrm{E} \exp \left(-\sqrt{2 \mathrm{t}} \mathrm{Y}_{0}\right), \quad \mathrm{t}>0
$$

Nous allons faire appel à la relation (80) reliant le pont brownien à la fonction spectrale empirique pour établir le résultat suivant.

THÉORÈmE 5. On a

$$
\lim _{t \rightarrow+\infty} t^{-1 / 3} \log E \exp \left(-\sqrt{2 t} Y_{0}\right)=-\left(27 \pi^{2} j_{0}^{2}\right)^{1 / 3}
$$

Preuve. Tout d'abord, en vertu de (80) on a

$$
\left(1 / 4 \pi^{2} \mathrm{t}\right) \mathrm{E} \exp \left(-\sqrt{2 \mathrm{t}} \mathrm{Y}_{0}\right)=\Phi(\mathrm{t}) \geq \tilde{E} \exp \left(-\mathrm{t} \lambda_{1}\right)
$$


d'où, avec (67),

$$
\begin{aligned}
\liminf _{t \rightarrow+\infty} t^{-1 / 3} \log E \exp \left(-\sqrt{2 t} Y_{0}\right) & \geq \lim _{t \rightarrow+\infty} t^{-1 / 3} \log \tilde{E} \exp \left(-t \lambda_{1}\right) \\
& =-\left(27 \pi^{2} j_{0}^{2}\right)^{1 / 3} .
\end{aligned}
$$

Pour établir la majoration inverse, rappelons que, d'une manière générale, si $\lambda_{n}, \mathrm{n} \geq 1$, désignent les valeurs propres (ordonnées en croissant) d'un domaine $U$, borné, quel conque, alors on a

$$
0<\lambda_{1}<\lambda_{2} \leq \cdots \leq \lambda_{\mathrm{n}} \leq \cdots ;
$$

et, en vertu d'un résultat de Li et Yau [13],

$$
\lambda_{\mathrm{n}} \geq 2 \mathrm{n} \pi / \mathrm{V}(\mathrm{U}), \quad \mathrm{n} \geq 2 .
$$

On déduit de (84) et (85) que la fonction spectrale empirique se majore selon

$$
\begin{aligned}
\Phi(\mathrm{t}) \leq & \tilde{\mathrm{E}} \exp \left(-\mathrm{t} \lambda_{1}\right)+\sum_{\mathrm{n}=2}^{\mathrm{N}-1} \tilde{\mathrm{E}} \exp \left(-\mathrm{t} \lambda_{\mathrm{n}}\right) \\
& +\tilde{\mathrm{E}}\left\{\frac{\exp (-2 \mathrm{~N} \pi / \mathrm{V})}{1-\exp (-2 \mathrm{t} \pi / \mathrm{V})}\right\} \quad \text { pour tout } \mathrm{N} \geq 3 .
\end{aligned}
$$

On voit donc, avec (84), que pour conclure il suffit d'établir qu'il existe $N \geq 3$, vérifiant

$$
\limsup _{t \rightarrow+\infty} t^{-1 / 3} \log \tilde{E}\left\{\frac{\exp (-2 N \mathrm{t} \pi / \mathrm{V})}{1-\exp (-2 \mathrm{t} \pi / \mathrm{V})}\right\} \leq-\left(27 \pi^{2} \mathrm{j}_{0}^{2}\right)^{1 / 3} .
$$

Notons, à cet effet, que

$$
\begin{aligned}
& \tilde{\mathrm{E}}\left\{\frac{\exp (-2 \mathrm{Nt} \pi / \mathrm{V})}{1-\exp (-2 \mathrm{t} \pi / \mathrm{V})}\right\} \\
& \leq\left(\frac{1}{\pi \mathrm{t}}\right) \tilde{\mathrm{E}}\left\{\left(\mathrm{V}^{2}+2 \pi \mathrm{t}\right) \exp \left(-\frac{2 \mathrm{Nt} \pi}{\mathrm{V}}\right)\right\} \\
& =\left(\frac{1}{\pi \mathrm{t}}\right) \int_{0}^{+\infty}\left(2 \mathrm{x}+2 \mathrm{Nt} \pi+\frac{4 \mathrm{~N} \pi^{2} \mathrm{t}^{2}}{\mathrm{x}^{2}}\right) \exp \left(-\frac{2 \mathrm{Nt} \pi}{\mathrm{x}}\right) \tilde{\mathrm{P}}\{\mathrm{V} \geq \mathrm{x}\} \mathrm{dx} \\
& \leq \frac{\mathrm{A}(\varepsilon)}{\pi \mathrm{t}} \int_{0}^{+\infty}\left(2 \mathrm{x}+2 \mathrm{Nt} \pi+\frac{4 \mathrm{~N} \pi^{2} \mathrm{t}^{2}}{\mathrm{x}^{2}}\right) \exp \left(-2(1-\varepsilon) \sqrt{\pi \mathrm{x}}-\frac{2 \mathrm{Nt} \pi}{\mathrm{x}}\right) \mathrm{dx} \text {. }
\end{aligned}
$$

La dernière majoration résultat, à nouveau, du Théorème 1 . On termine facilement, en faisant appel, une nouvelle fois, à la méthode de Laplace.

\section{Comportement asymptotique du périmètre de l'enveloppe con-} vexe de la trajectoire du mouvement brownien plan. Nous allons montrer que (81) reste valable en remplaçant le périmètre $Y_{0}$ de la trajectoire du pont brownien par celui de la trajectoire du mouvement brownien. 
Considérons donc un mouvement brownien plan,

(89) $\mathrm{W}(\mathrm{s}), \quad 0 \leq \mathrm{s} \leq \mathrm{t}, \quad \mathrm{W}(0) \equiv 0, \quad \mathrm{t}>0 \quad$ démarrant de l'origine.

Soient

$$
\mathbf{W}_{\mathrm{t}}=\{\mathrm{W}(\mathrm{s}), 0 \leq \mathrm{s} \leq \mathrm{t}\}, \quad \mathrm{t}>0,
$$

les trajectoires associées puis $Y$ le périmètre de l'enveloppe convexe de $\mathbf{W}_{1}$. Nous allons établir le théorème suivant.

THÉORÈME 6. On a

$$
\lim _{t \rightarrow+\infty} t^{-1 / 3} \log E \exp (-\sqrt{2 t} Y)=-\left(27 \pi^{2} j_{0}^{2}\right)^{1 / 3} .
$$

Preuve. Soient $D_{i}, i=1 \ldots N_{R}$, les domaines polygonaux convexes, associés à la mesure de Poisson aléatoire $P=\left\{x_{n}, n \geq 1\right\}$ et inclus dans le disque ouvert $\mathrm{B}(\mathrm{R})$. Comme au chapitre précédent, désignons par $\lambda_{n, i}, \mathrm{n} \geq 1$, les valeurs propres du laplacien $\Delta=\partial^{2} / \partial \mathbf{x}^{2}+\partial^{2} / \partial \mathbf{y}^{2}$, pour le problème de Dirichlet sur le domaine $D_{i}$, et par $\phi_{n, i}$ les fonctions propres associées (normalisées en norme $L^{2}$ ). Notons

$$
\varphi_{i}(t)=\sum_{n=1}^{+\infty} \exp \left(-t \lambda_{n, i}\right)\left[\int_{D_{i}} \phi_{n, i} d x\right]^{2}, \quad t>0,
$$

et soit alors

$$
\varphi_{\mathrm{R}}(\mathrm{t})=\left(1 / \mathrm{N}_{\mathrm{R}}\right) \sum_{\mathrm{i}=1}^{\mathrm{N}_{\mathrm{R}}} \varphi_{\mathrm{i}}(\mathrm{t}) .
$$

En reprenant la démonstration du Théorème 1 de [6], on démontre facilement avec [11] (par la même méthode) le théorème.

THÉORÈME 7. Presquesûrement, pour tout $\mathrm{t}>0$, la suite $\varphi_{\mathrm{R}}(\mathrm{t})$ admet, Iorsque $\mathrm{R} \rightarrow+\infty$, une limite détermi niste finie $\varphi(\mathrm{t}) \mathrm{s}^{\prime}$ exprimant sous la forme

$$
\begin{aligned}
\varphi(\mathrm{t}) & =(1 / \pi) \mathrm{E} \exp (-\sqrt{2 \mathrm{t}} \mathrm{Y}) \\
& =(1 / \pi) \mathrm{P}\left\{\mathbf{W}_{2 \mathrm{t}} \subset \mathrm{D}_{0}\right\} .
\end{aligned}
$$

Notons maintenant, les majorations triviales,

$$
\begin{aligned}
\left(\int_{D_{i}} \phi_{n, i} d x\right)^{2} & \leq\left[\int_{D_{i}}\left(\phi_{n, i}\right)^{2} d x\right] V\left(D_{i}\right) \\
& =V\left(D_{i}\right), \quad 1 \leq i \leq N_{R} \text { et } n \geq 1 .
\end{aligned}
$$


Nous en déduisons, par une démarche identique à celle de la preuve du Théorème 5 et faisant appel à (85) que

$$
\begin{aligned}
(1 / \pi) E \exp (-\sqrt{2 \mathrm{t} Y}) \leq & \tilde{E} \exp \left(-\mathrm{t} \lambda_{1}\right)+\sum_{\mathrm{n}=2}^{\mathrm{N}-1} \tilde{E} \exp \left(-\mathrm{t} \lambda_{\mathrm{n}}\right) \\
& +\tilde{E}\left\{\frac{\exp (-2 \mathrm{Nt} \pi / \mathrm{V})}{1-\exp (-2 \mathrm{t} \pi / \mathrm{V})}\right\}, \quad \mathrm{N} \geq 3 .
\end{aligned}
$$

En faisant appel à (16), on en déduit comme précédemment que

$$
\limsup _{\mathrm{t} \rightarrow+\infty} \mathrm{t}^{-1 / 3} \log \tilde{E}\left\{\frac{\exp (-2 \mathrm{Nt} \pi / \mathrm{V})}{1-\exp (-2 \mathrm{t} \pi / \mathrm{V})}\right\} \leq-\left(27 \pi^{2} \mathrm{j}_{0}^{2}\right)^{1 / 3},
$$

d'où il résulte, avec (67) et (84), que

$$
\begin{aligned}
\limsup _{t \rightarrow+\infty} t^{-1 / 3} \log E \exp (-\sqrt{2 t} Y) & \leq \lim _{\mathrm{t} \rightarrow+\infty} \mathrm{t}^{-1 / 3} \log \tilde{E} \exp \left(-\mathrm{t} \lambda_{1}\right) \\
& =-\left(27 \pi^{2} \mathrm{j}_{0}^{2}\right)^{1 / 3}
\end{aligned}
$$

II nous reste à voir que l'on a

$$
\begin{aligned}
\liminf _{\mathrm{t} \rightarrow+\infty} \mathrm{t}^{-1 / 3} \log \mathrm{E} \exp (-\sqrt{2 \mathrm{t} Y}) & \geq \lim _{\mathrm{t} \rightarrow+\infty} \mathrm{t}^{-1 / 3} \log \tilde{E} \exp \left(-\mathrm{t} \lambda_{1}\right) \\
& =-\left(27 \pi^{2} \mathrm{j}_{0}^{2}\right)^{1 / 3}
\end{aligned}
$$

Pour cela, nous allons utiliser la majoration suivante, due à Payne et Rayner (voir [12], par exemple):

$$
\left(\int_{U} \phi_{1} \mathrm{dx}\right)^{2} \geq \frac{4 \pi}{\lambda_{1}}\left[\int_{U}\left(\phi_{1}\right)^{2} \mathrm{dx}\right]
$$

satisfaite pour tout domaine borné $U$ du plan, $\lambda_{1}$ et $\phi_{1}$ désignant respectivement la première valeur propre et la première fonction propre du Laplacien (pour le problème de Dirichlet) du domaine U.

II résulte de (92)-(94) et (100), que

$$
\begin{aligned}
(1 / \pi) \mathrm{E} \exp (-\sqrt{2 \mathrm{t} Y}) & \geq \lim _{\mathrm{R} \rightarrow+\infty} \frac{1}{\mathrm{~N}_{\mathrm{R}}} \sum_{\mathrm{i}=1}^{\mathrm{N}_{\mathrm{R}}} \frac{4 \pi}{\lambda_{1, \mathrm{i}}} \exp \left(-\mathrm{t} \lambda_{1, \mathrm{i}}\right) \\
& =4 \pi \tilde{\mathrm{E}}\left[\frac{\exp \left(-\mathrm{t} \lambda_{1}\right)}{\lambda_{1}}\right] \\
& \geq 4 \pi \tilde{\mathrm{E}}\left[\frac{\exp \left(-\mathrm{t} \mu_{1}\right)}{\mu_{1}}\right] \\
& \geq 4 \pi \int_{0}^{+\infty} \frac{\mathrm{x}^{2}}{\mathrm{j}_{0}^{2}} \exp \left\{-2 \pi \mathrm{x}-\frac{\mathrm{tj}_{0}^{2}}{\mathrm{x}^{2}}\right\} \mathrm{dx} .
\end{aligned}
$$

La dernière majoration se déduisant de (12). On en tire facilement la propriété (99) souhaitée (via, à nouveau, la méthode de Laplace). 
REMARQUE. Le Théorème 6 présente une certaine parenté avec le résultat classique de Donsker-Varadhan [4] précisant le comportement asymptotique du volume de la saucisse de Wiener. Rappelons, en effet, qu'en prenant un modèle booléen $M(\alpha)$ (voir [10]) admettant pour forme générique une boule de rayon $\alpha>0$ fixé, les centres des boules constituant une mesure de Poisson aléatoire admettant pour mesure d'intensité la mesure de Lebesque de l'espace $\mathrm{R}^{2}$, puis en désignant par $\mathrm{D}_{0}(\alpha)$ la composante connexe de l'origine du modèle booléen [avec la convention $\mathrm{D}_{0}(\alpha)=\varnothing$ lorsque l'origine est recouverte par les boules] on a

$$
\mathrm{P}\left\{\mathbf{W}_{\mathrm{t}} \subset \mathrm{D}_{0}(\alpha)\right\}=\exp \left(-\int_{\mathbf{W}(\mathrm{t}, \alpha)} \mathrm{dx}\right)
$$

où

$$
\mathbf{W}(\mathrm{t}, \alpha)=\mathbf{W}_{\mathrm{t}}+\mathrm{B}(\alpha), \quad \mathrm{t}>0,
$$

est la saucisse de Wiener. Donsker et Varadhan ont démontré (voir [4]), par une technique des grandes déviations, que

(104) $\lim _{\mathrm{t} \rightarrow+\infty} \mathrm{t}^{-1 / 2} \log \mathrm{E}\left\{-\int_{\mathbf{w}(\mathrm{t}, \alpha)} \mathrm{dx}\right\}=-\left(2 \pi \mathrm{j}_{0}^{2}\right)^{1 / 2}, \quad \alpha>0$ étant fixé.

On sait d'ailleurs, en vertu d'un résultat de Hall [9], que le processus poissonien de droites peut s'obtenir à partir des modèles booléens $M(\alpha)$, $\alpha>0$, par un passage à la limite. Plus précisément, le domaine (aléatoire) $\alpha \mathrm{D}_{0}(\alpha)$ converge en loi (conditionnellement au fait que l'origine n'est pas recouverte par les boules) lorsque $\alpha \rightarrow+\infty$, vers la cellule de Crofton $D_{0}$. Cela étant, le résultat asymptotique (19) ne se déduit pas de (104) et la technique de [4] ne nous semble pas pouvoir permettre d'y accéder.

6. La contrepartie brownienne à la conjecture de D. G. Kendall. Rappelons, tout d'abord, le résultat classique suivant de grandes déviations (voir [4], par exemple).

\section{THÉORÈME 8. Désignons par}

$$
M(t)=\sup _{0 \leq s \leq t}\|W(s)\|
$$

le maximum de la composante radiale du mouvement brownien plan.

On a alors

$$
\lim _{\mathrm{t} \rightarrow+\infty} \mathrm{t}^{-1 / 3} \log \mathrm{E} \exp (-2 \pi \sqrt{2 \mathrm{t}} \mathrm{M}(1))=-\left(27 \pi^{2} \mathrm{j}_{0}^{2}\right)^{1 / 3} .
$$

Fixons $0<\varepsilon<1$, puis considérons les probabilités conditionnelles

$$
\mathrm{P}\{\mathrm{Y} \geq(1-\varepsilon) 2 \pi \mathrm{a} \mid \mathrm{M}(1)=\mathrm{a}\}
$$

et

$$
\mathrm{P}\left\{\mathbf{W}_{1} \supset \mathrm{B}((1-\varepsilon) \mathrm{a}) \mid \mathrm{M}(1)=\mathrm{a}\right\} .
$$


On voit facilement, par un argument géométrique évident, que

$$
\begin{aligned}
& \underset{a \rightarrow 0}{\limsup } P\{Y \geq(1-\varepsilon) 2 \pi a \mid M(1)=a\}=1 \\
& \quad \Leftrightarrow \limsup _{a \rightarrow 0} P\left\{\mathbf{W}_{1} \supset B((1-\varepsilon) a) \mid M(1)=a\right\}=1 .
\end{aligned}
$$

Nous allons établir le théorème suivant.

THÉORÈME 9. Pour tout $0<\varepsilon<1$, fixé, on a

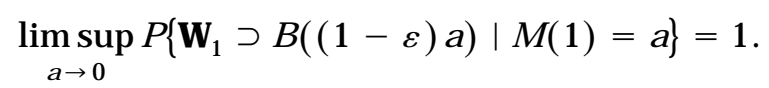

Preuve du Théorème 9. En effet, dans le cas contraire, il existerait $\mathrm{a}_{0}>0$ et $\varepsilon>0$ vérifiant

(111) $\mathrm{P}\{\mathrm{Y} \geq(1-\varepsilon) 2 \pi \mathrm{a} \mid \mathrm{M}(1)=\mathrm{a}\} \leq 1-\varepsilon$ pour tout $0<\mathrm{a}<\mathrm{a}_{0}$, soit encore

(112) $\mathrm{P}\{\mathrm{Y} \leq(1-\varepsilon) 2 \pi \mathrm{a} \mid \mathrm{M}(1)=\mathrm{a}\}>\varepsilon$ pour tout $0<\mathrm{a}<\mathrm{a}_{0}$.

Désignons par $\mathrm{M}(\mathrm{P})$ la loi de la variable aléatoire $\mathrm{M}(1)$. On a alors

$$
\begin{aligned}
\int \exp (-\sqrt{2 \mathrm{t} Y}) \mathrm{dP} \geq & \int_{\left\{Y \leq(1-\varepsilon) 2 \pi \mathrm{M}(1), \mathrm{M}(1) \leq \mathrm{a}_{0}\right\}} \exp (-\sqrt{2 \mathrm{t} Y}) \mathrm{dP} \\
\geq & \int_{\left\{\mathrm{Y} \leq(1-\varepsilon) 2 \pi \mathrm{M}(1), \mathrm{M}(1) \leq \mathrm{a}_{0}\right\}} \exp \{-\sqrt{2 \mathrm{t}}(1-\varepsilon) 2 \pi \mathrm{M}(1)\} \mathrm{dP} \\
= & \int_{0}^{\mathrm{a}_{0}} \mathrm{P}\{\mathrm{Y} \leq(1-\varepsilon) 2 \pi \mathrm{a} \mid \mathrm{M}(1)=\mathrm{a}\} \\
& \times \exp \{-\sqrt{2 \mathrm{t}}(1-\varepsilon) 2 \pi \mathrm{a}\} \mathrm{dM}(\mathrm{P})(\mathrm{a}) \\
\geq & \varepsilon \int_{\left\{\mathrm{M}(1) \leq \mathrm{a}_{0}\right\}} \exp \{-\sqrt{2 \mathrm{t}}(1-\varepsilon) 2 \pi \mathrm{M}(1)\} \mathrm{dP}
\end{aligned}
$$

II en résulte que

$$
\begin{aligned}
& \lim _{t \rightarrow+\infty} t^{-1 / 3} \log E \exp (-\sqrt{2 t} Y) \\
& \geq \limsup _{t \rightarrow+\infty} t^{-1 / 3} \log \left[\int_{\left\{M(1) \leq a_{0}\right\}} \exp \{-\sqrt{2 t}(1-\varepsilon) 2 \pi M(1)\} d P\right]
\end{aligned}
$$

Par ailleurs, on a trivialement

(115) $\lim _{\mathrm{t} \rightarrow+\infty} \mathrm{t}^{-1 / 3} \log \left[\int_{\left\{\mathrm{M}(1) \geq \mathrm{a}_{0}\right\}} \exp \{-\sqrt{2 \mathrm{t}}(1-\varepsilon) 2 \pi \mathrm{M}(1)\} \mathrm{dP}\right]=-\infty$ et donc d'après (106),

$$
\begin{gathered}
\limsup _{t \rightarrow+\infty} t^{-1 / 3} \log \left[\int_{\left\{M(1) \leq a_{0}\right\}} \exp \{-\sqrt{2 t}(1-\varepsilon) 2 \pi M(1)\} d P\right] \\
=-\left\{27 \pi^{2} j_{0}^{2}(1-\varepsilon)^{2}\right\}^{1 / 3} .
\end{gathered}
$$


Les relations (114), (116) et (91) apportent, alors, la contradiction souhaitée.

Remarque. II est possible de montrer que

$$
\lim _{\mathrm{a} \rightarrow 0} \mathrm{P}\left\{\mathbf{W}_{1} \supset \mathrm{B}((1-\varepsilon) \mathrm{a}) \mid \mathrm{M}(1)=\mathrm{a}\right\}=1 .
$$

Cette propriété qui nécessite une étude fine des probabilités conditionnelles,

$$
P\{Y \leq b \mid M(1)=a\}, \quad b>0, a>0,
$$

fera l'objet d'une publication ultérieure.

\section{RÉFÉRENCES}

[1] Bonnesen, T. and Fenchel, W. (1971). Theorie der Konvexen Körper. Chelsea, New York.

[2] Cowan, R. (1978). The use of the ergodic theorems in random geometry. Suppl. Adv. Appl. Probab. 10 47-57.

[3] Cowan, R. (1980). Properties of ergodic random mosaic processes. Math. Nachr. 97 89-102.

[4] Donsker, M. D. and Varadhan, S. R. S. (1975). Asymptotics for the Wiener sausage. Comm. Pure Appl. Math. 27 525-565.

[5] George, E. I. (1987). Sampling random polygons. Suppl. J . Appl. Probab. 24 557-573.

[6] Goldman, A. (1996). Le spectre de certaines mosaïques poissoniennes du plan et l'enveloppe convexe du pont brownien. Probab. Theory Related Fields 105 57-83.

[7] Goldman, A. (1998). Sur une conjecture de D. G. Kendall concernant la cellule de Crofton du plan et sur sa contrepartie brownienne. Comptes Rendus Acad. Sci. Paris 326 233-237.

[8] Goudsmit, S. A. (1945). Random distributions of lines in a plane. Rev. Modern Phys. 17 $321-322$.

[9] HALL, P. (1985). Distribution of size, structure and number of vacant regions in a high intensity mosaic. Z. Warsch. Verw. Gebiete 70 237-261.

[10] HaLL, P. (1988). Introduction to the Theory of Coverage Processes. Wiley, New York.

[11] KAC, M. (1966). Can one hear the shape of a drum? Amer. Math. Monthly 73 1-23.

[12] Kuttler, J . R. and Sigilitto, V. G. (1984). Eigenvalues of the Laplacian in two dimensions. SIAM Rev. 26 163-193.

[13] LI, P. and YAU, S. T. (1983). On the Schrödinger equation and the eigenvalue problem. Comm. Math. Phys. 88 309-318.

[14] Magnus, W., Oberhettinger, F. and Soni, R. P. (1966). Formulas and Theorems for the Special Functions of Mathematical Physics. Springer, New York.

[15] Matheron, G. (1975). Random Sets and Integral Geometry. Wiley, New York.

[16] Miles, R. E. (1964). Random polygons determined by random lines in a plane I. Proc. Nat. Acad. Sci. U.S.A. 52 901-907.

[17] MILES, R. E. (1964). Random polygons determined by random lines in a plane II. Proc. Nat. Acad. Sci. U.S.A. 52 1157-1160.

[18] Miles, R. E. (1973). The various aggregates of random polygons determined by random lines in a plane. Adv. in Math. 10 256-290.

[19] MILES, R. E. (1995). A heuristic proof of a long-standing conjecture of D. G. Kendall concerning the shapes of certain large random polygons. Adv. in Appl. Probab. 27 397-417.

[20] Paroux, K. (1998). Quelques théorèmes centraux limites pour les processus poissoniens de droites dans le plan. Adv. in Appl. Probab. A paraître.

[21] PólyA, G. and Szegö, G. (1951). I soperimetric Inequalities in Mathematical Physics. Princeton Univ. Press.

[22] Solomon, H. (1978). Geometric Probability. SIAM, Philadelphia.

[23] Stoyan, D., Kendall, W. S. and MeCKE, J . (1987). Stochastic Geometry and Its Applications. Wiley, New York. 
[24] TANnER, J . C. (1983). The proportion of quadrilaterals formed by random lines in a plane. J. Appl. Probab. 20 400-404.

[25] TAnner, J. C. (1984). Polygons formed by random lines in a plane: some further results. J. Appl. Probab. 20 778-787.

[26] Wiener, N. (1939). The ergodic theorem. Duke Math J . 5 1-18.

Laboratoire de Probabilités UFR DE MATHÉMATIQUES Université CLAUde BeRnaRd Lyon 1 43, BOULEVARD DU 11 NOVEMBRE 1918 F-69622 VILLEURBANNE CEDEX FRANCE

E-MAIL: goldman@jonas.univ-Iyon1.fr 